\title{
Comparisons of Masticatory Movements between Mixing Ability Test and Masticatory Performance Test
}

\author{
Kenji Fueki, DDS, PhD, Eiko Yoshida, DDS, and Yoshimasa Igarashi, DDS, PhD \\ Removable Partial Denture Prosthodontics, Graduate School, Tokyo Medical and Dental University, Tokyo, \\ Japan
}

\section{Clinical significance}

In application of the mixing ability test for evaluating objective masticatory function, clinicians should recognize that the mixing ability test is partially different from a traditional masticatory performance test in respect of masticatory movements.

\begin{abstract}
Purpose: The purpose of this study was to compare mandibular movements in the mixing ability test with those in the masticatory performance test.

Methods: Twenty-six dentate subjects (mean age 25.3 years) performed a mixing ability test using a twocolored paraffin wax cube and a masticatory performance test using $3 \mathrm{~g}$ peanuts. Mandibular movements during the chewing of each test item were recorded using an opto-electronic system. Twelve parameters of mandibular movements on a mandibular incisal point were measured for each masticatory cycle. The mean from $2^{\text {nd }}$ to $5^{\text {th }}$ cycle in each parameter was employed for paired $t$-test.

Results: 7 of 12 parameters showed significant mean differences between the mixing ability test and the masticatory performance test. Vertical amplitude, anteroposterior amplitude, maximum closing velocity and opening angle for the mixing ability test were significantly greater than for the masticatory performance test $(P<0.05)$. Opening duration and cycle duration for the mixing ability test were significantly longer than for the masticatory performance test $(P<0.05)$. Envelope angle for the mixing ability test was significantly less than for the masticatory performance test $(P<0.001)$.
\end{abstract}

Conclusion: The results of this study suggest mandibular movements in the mixing ability test are partially different from those in masticatory performance test.

\section{Corresponding to: Dr Kenji Fueki}

Removable Partial Denture Prosthodontics, Graduate

School, Tokyo Medical and Dental University

1-5-45 Yushima, Bunkyo-ku, Tokyo 113-8549, Japan

Tel: +81-3-5803-5514, Fax: +81-3-5803-5514

E-mail: kunfu.rpro@tmd.ac.jp

Received on April 18, 2007 / Accepted on June 13, 2007
Key words: mixing ability test, masticatory performance test, masticatory movement

\section{Introduction}

Recovery of impaired masticatory function due to tooth loss is one of the purposes of prosthodontic treatment. A variety of methods have been utilized for evaluating objective masticatory function. ${ }^{1}$ The masticatory performance test is the most common method which allows an estimate of ability to comminute food based on particle size distribution of food bolus. ${ }^{2-4}$ Natural foods such as peanuts and raw carrot, ${ }^{2,3}$ and artificial materials ${ }^{4}$ having hard and brittle properties, have been commonly employed as test items for masticatory performance tests.

More recently, chewing tests using chewing gum $^{5,6}$ or paraffin wax $^{7}$ as a test item have been developed. Since test items used for the newly developed chewing tests have soft and cohesive properties, they seem to evaluate ability of bolus shaping and kneading. Although the masticatory performance test was the most common method, the analysis is complicated for routine clinical evaluation. Therefore, we have developed the mixing ability test to evaluate masticatory function more easily at chair side. The mixing ability test utilizes a wax cube made of two-colored paraffin wax. Mixing ability index (MAI) is determined from color mixture and shape of a chewed wax cube. A previous study has found moderate correlations between MAI and comminuting ability index determined with a masticatory performance test, ${ }^{8}$ suggesting food mixing ability is moderately related to food comminuting ability.

Mandibular movements while chewing various test foods (masticatory movements) have been utilized for evaluating masticatory function of patients with dental prostheses. ${ }^{9-11}$ It is widely known that a central pattern generator located in the brain stem is primarily responsible for 


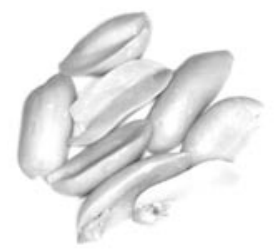

a

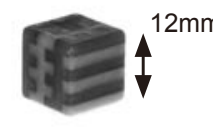

b

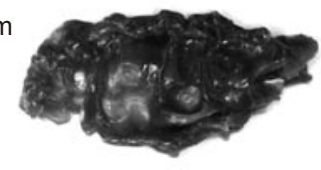

C
Fig. $13 \mathrm{~g}$ peanuts used for the masticatory performance test (a), wax cube used in the mixing ability test before chewing (b), an example after chewing for 10 strokes (c).

rhythmic chewing pattern. ${ }^{12}$ Animal studies have demonstrated the chewing pattern can be modified by sensory feedback from periodontal receptors and muscle spindles. ${ }^{13,14}$ A number of studies have demonstrated that masticatory movement patterns in dentate subjects are closely related to the type of natural foods, ${ }^{15,16}$ hardness ${ }^{17,18}$ and size $^{19}$ of test item. These suggest that sensory feedback in respect of food properties is responsible for specific masticatory movement patterns. The shape and size of the wax cube used for the mixing ability test is different from those of the peanuts used for the masticatory performance test (Fig. 1). The wax cube has a cohesive property, thus it maintained a single bolus during mastication. The hardness of the wax cube is less than that of a peanut or raw carrot used for the masticatory performance test. ${ }^{20}$ A recent study has shown moderate association between mixing ability index and masticatory movements during chewing of a wax cube. ${ }^{21}$ However, it is not clear how the differences of test items between the mixing ability test and the masticatory performance test affect the masticatory movement pattern.

The purpose of this study was to clarify differences in masticatory movements between the mixing ability test and the masticatory performance test. The null hypothesis to be tested in this study was that no parameters in masticatory movements show mean differences between the mixing ability test and the masticatory performance test.

\section{Materials and methods}

\section{Subjects}

The 26 participants in this study were completely natural dentition with Angle Class I molar relationship and balanced for gender (13 males, 13 females, mean age $25.3 \pm 2.2$ years). Subjects were recruited from the students of Tokyo Medical and Dental University. Applicants with severe periodontal disease, history of orthodontic treatment in the past three years, and clinical signs or symptoms of temporomandibular disorder and salivary dysfunction were excluded. Each subject received a written and oral description of the study, and informed consent was obtained prior to enrollment into the study. All study related procedures and tests were approved by the Ethical Committee at Tokyo Medical and Dental University (No. 229).

\section{Mixing ability test and masticatory performance test}

A mixing ability test ${ }^{7}$ and a masticatory performance test ${ }^{3}$ were performed in this study. For the mixing ability test, the subjects were directed to chew a two-colored (red/green) paraffin wax cube (12-mm cube) for 10 strokes on their preferred chewing side (Fig. 1). When the preferred chewing side was not clear, the right side was indicated. The wax cube was maintained in an incubator at $37^{\circ} \mathrm{C}$ until chewing. Subjects completed two adaptation trials, chewing two sample cubes separately, with full setting of recording of masticatory movements. Following the adaptation trials, three experimental trials were given (one wax cube at a time). Then, a standardized masticatory performance test was given to the subjects. ${ }^{3}$ Three test portions, 3 grams each, were employed as a test item. The subjects were asked to chew peanuts for 20 strokes on the same side of the mouth as for the mixing ability test.

\section{Recordings and quantification of masticatory movements}

Recording of masticatory movements during each chewing test and analysis of masticatory movements were according to a method in a previous study. ${ }^{21}$ A six-degrees-of freedom opto-electronic system (Gnatho-hexagraph system Ver. 1.31, Ono Sokki Co., Ltd., Kanagawa, Japan) was used to measure three-dimensional movements of an arbitrary point on the mandible. A face bow (12 g) with three LEDs was attached to the labial surface of the lower incisors with a crutch, and a head frame $(170 \mathrm{~g})$ with three LEDs was placed on the head parallel to the Frankfort horizontal plane using a headband. The three-dimensional positions of each of the six LEDs were measured using two CCD cameras placed in front of the subject. The three-dimensional position of a man- 
dibular central incisor point was recorded as a reference point using a pointer with two LEDs prior to masticatory tests. Subjects were seated comfortably in an upright position in a dental chair in an electrically shielded room. Each test item was placed on the tongue of each subject, and subjects were asked to perform maximum intercuspation. This position was recorded as centric occluding ( $\mathrm{CO}$ ) position. Recording of mandibular movements started when subjects performed maximum intercuspation, and the recording was terminated when subjects finished the last chewing stroke.

The measured data for each masticatory test were digitized at $89.4 \mathrm{~Hz}$ and stored in a personal computer for subsequent analysis. Commercial software (Gnatho-analyzer, Ono Sokki Co., Ltd., Kanagawa, Japan) computed three-dimensional movements of the mandibular central incisor point, and automatically identified each chewing cycle. When the mandible did not return to CO position in the closing phase, the cycle was excluded from the analysis. Then, the identified chewing cycle was broken into three (open-closeocclusal) phases. Vertical displacement of $0.7 \mathrm{~mm}$ below CO position was employed as a slice level to determine occlusal phase. Opening duration (s), closing duration (s), occlusal duration (s) and cycle duration $(\mathrm{s})$ were determined for each chew ${ }^{-}$ ing cycle (Fig. 2). Then, vertical amplitude ( $\mathrm{mm}$ ), lateral amplitude $(\mathrm{mm})$, anteroposterior (AP) amplitude $(\mathrm{mm})$, were computed for each chewing cycle (Fig. 3). Velocity was computed with firstordered derivation of vertical displacement, and maximum velocities $\left(\mathrm{mm} \mathrm{s}^{-1}\right)$ were determined in the opening phase and closing phase for each chewing cycle. Opening and closing angles (degrees) were determined at a level $0.5 \mathrm{~mm}$ below $\mathrm{CO}$ position in the frontal plane. ${ }^{22}$ Envelope angle was determined at a turning point to the closing path from the opening path in the frontal plane (Fig. 4). ${ }^{10}$ The mean of the three trials for each chewing test was used for statistical analysis.

\section{Statistical analysis}

The normality of each measured variable was tested using the Kolmogorov-Smirnov test $(P>0.15)$. Thus, parametric tests were employed for statistical analysis. According to a recommendation to achieve the best discrimination of masticatory movements for different test items ${ }^{18}$, the mean of cycles \#2-5 was employed for statistical comparisons. A paired $t$-test was used to test the

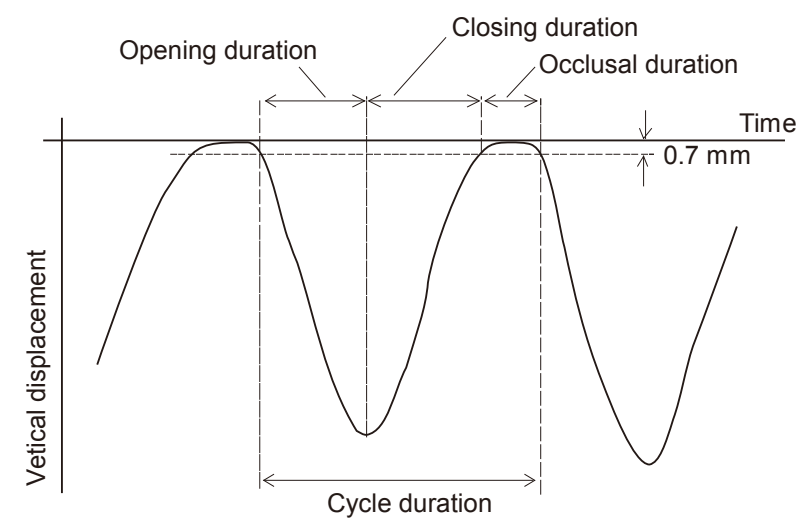

Fig. 2 Schematic representation of opening duration, closing duration, occlusal duration, and cycle duration.
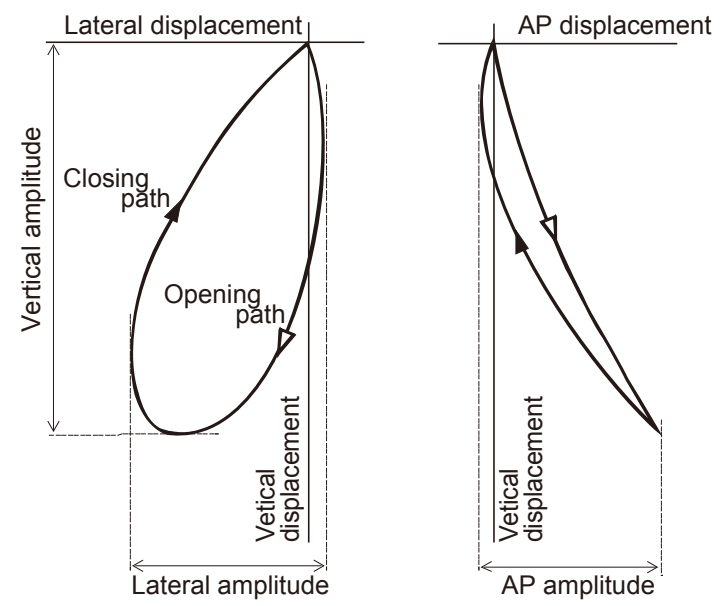

Fig. 3 Schematic representation of vertical amplitude, AP amplitude, lateral amplitude, in the frontal or sagittal plane.

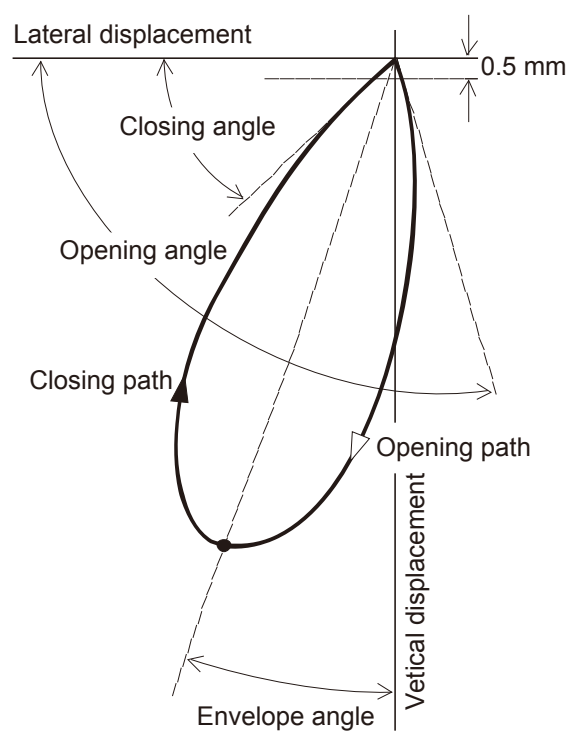

Fig. 4 Schematic representation of opening angle, closing angle and envelope angle of a masticatory envelope in the frontal plane. 
mean difference in each parameter between the mixing ability test and the masticatory performance test. SPSS 11.5 J (SPSS Japan Inc, Tokyo, Japan) was used for the statistical analyses. $P$-values less than 0.05 were considered significant.

\section{Results}

Examples of masticatory movements for the mixing ability test and the masticatory performance test are shown in Figure 5. Mean and standard deviation (s.d.) for each parameter for each chewing test are shown in Table 1 . In 7 of 12 parameters of masticatory movements, significant mean differences between the mixing ability test and the masticatory performance test were found $(P<0.05)$. Vertical amplitude, AP amplitude, maximum closing velocity and opening angle for the mixing ability test were significantly greater than for the masticatory performance test $(P<0.05)$. Opening duration and cycle duration for the mixing ability test were significantly longer than for the masticatory performance test $(P<0.05)$. Envelope angle for the mixing ability test was significantly less than for the masticatory performance test $(P<0.001)$. The remaining 5 parameters, lateral amplitude, maximum opening velocity, closing duration, occlusal duration and closing angle did not show any significant mean differences $(P>0.12)$.

\section{Discussion}

In 4 of 12 parameters of masticatory movements (vertical amplitude, AP amplitude, opening duration and envelope angle), highly significant mean differences were found between the mixing ability test and the masticatory performance test $(P<0.001)$ (Table 1). Furthermore, 3 parameters (maximum closing velocity, cycle duration and opening angle) showed significant mean differences $(P<0.05)$. These results indicate that the masticatory movement pattern for the mixing ability test is partially different from that for the masticatory performance test. Thus, the null hypothesis in this study was rejected.

A variety of factors regarding food property have been found to affect masticatory movements. ${ }^{15-19}$ Sensory feedback from periodontal receptors and muscle spindles to the central

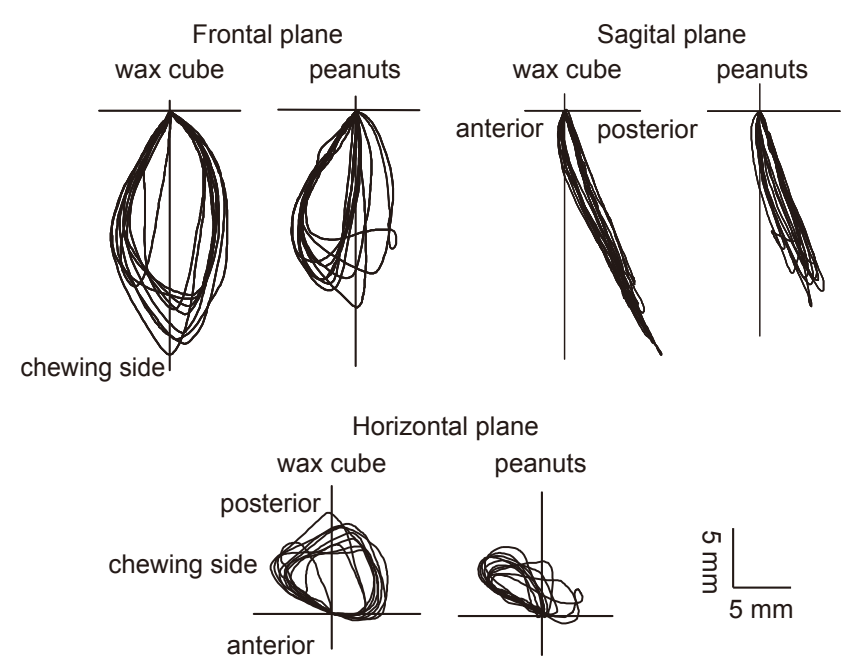

Fig. 5 Examples of masticatory movements during chewing a wax cube (mixing ability test) and peanuts (masticatory performance test). Chewing cycles $1^{\text {st }}$ to $10^{\text {th }}$ for each chewing test were superimposed in the each plane.

pattern generator is primarily responsible for modulation of chewing pattern. ${ }^{13,14}$ Therefore, two major factors, the difference in the size and the texture (hardness, cohesiveness, brittleness, adhesiveness) of the test items, are likely responsible for the differences in masticatory movement patterns between the two chewing tests. However, researchers have pointed out that a controlled study in size and texture of test items should be made to explain how each factor affects masticatory movements. ${ }^{18,23}$ Thus, this limitation should be taken into consideration for interpreting the results of the current study.

A study has shown maximum jaw gapes are related to the height of the food bolus. ${ }^{24}$ Thus, the size of test items is a possible factor accounting for differences of vertical amplitude between the two chewing tests. The initial thickness of the wax cube $(12 \mathrm{~mm})$ is greater than that of a single peanut (approximately $5 \mathrm{~mm}$ ). Thus, more interocclusal distance in molar teeth is necessary to bite a wax cube than for a single peanut. Indeed, mean difference of vertical amplitude between the mixing ability test and the masticatory performance test $(6.4 \mathrm{~mm})$ was similar to that of the test items $(7 \mathrm{~mm})$. AP amplitude is closely related to vertical amplitude because the incisal point of the mandible moves down in the inferior-posterior direction as shown in Figure 2. This may explain why AP amplitude showed the same trend as for vertical amplitude.

Maximum opening velocity was similar in the 
Table 1 Comparisons of masticatory movements between the mixing ability test and the masticatory performance test. The mean of cycles \#2-5 for each parameter was used.

\begin{tabular}{|c|c|c|c|c|c|c|c|}
\hline \multirow[t]{2}{*}{ Parameter } & \multicolumn{3}{|c|}{ Mixing ability test } & \multicolumn{3}{|c|}{$\begin{array}{c}\text { Masticatory } \\
\text { performance test }\end{array}$} & \multirow[t]{2}{*}{$P$ value } \\
\hline & Mean & \pm & s.d. & Mean & \pm & s.d. & \\
\hline Vertical amplitude $(\mathrm{mm})$ & 26.1 & \pm & 4.5 & 19.7 & \pm & 3.7 & $<0.001$ \\
\hline Lateral amplitude (mm) & 7.7 & \pm & 2.6 & 7.7 & \pm & 2.2 & 0.885 \\
\hline AP amplitude (mm) & 11.0 & \pm & 3.7 & 7.9 & \pm & 2.0 & $<0.001$ \\
\hline Max. opening velocity $\left(\mathrm{mm} \mathrm{s}^{-1}\right)$ & 139.0 & \pm & 29.8 & 131.5 & \pm & 33.1 & 0.118 \\
\hline Max. closing velocity $\left(\mathrm{mm} \mathrm{s}^{-1}\right)$ & 117.0 & \pm & 35.2 & 106.4 & \pm & 35.4 & 0.028 \\
\hline Cycle duration (s) & 1.03 & \pm & 0.17 & 0.94 & \pm & 0.17 & 0.034 \\
\hline Opening duration (s) & 0.36 & \pm & 0.08 & 0.26 & \pm & 0.05 & $<0.001$ \\
\hline Closing duration (s) & 0.43 & \pm & 0.09 & 0.45 & \pm & 0.11 & 0.578 \\
\hline Occlusal duration (s) & 0.24 & \pm & 0.07 & 0.23 & \pm & 0.05 & 0.354 \\
\hline Opening angle $\left({ }^{\circ}\right)$ & 97.0 & \pm & 33.1 & 90.1 & \pm & 28.5 & 0.042 \\
\hline Closing angle $\left(^{\circ}\right)$ & 52.1 & \pm & 11.0 & 51.3 & \pm & 11.2 & 0.693 \\
\hline Envelope angle $\left(^{\circ}\right)$ & 5.0 & \pm & 5.1 & 12.4 & \pm & 6.0 & $<0.001$ \\
\hline
\end{tabular}

two chewing tests. The vertical amplitude for the mixing ability tests was significantly greater than for the masticatory performance test. As a result, opening duration for the mixing ability test was longer than for the masticatory performance test. In contrast, maximum closing velocity for the mixing ability test was greater than for the masticatory performance test. Consequently, closing duration was similar for the two chewing tests. Since no significant differences between the two chewing tests were found in both closing duration and occlusal duration, the longer opening duration for the mixing ability test than for the masticatory performance test was responsible for the significant difference in cycle duration between the two chewing tests.

Envelope angle for the mixing ability test was less than for the masticatory performance test. This indicates masticatory movements during chewing of the peanuts showed more laterallyoriented chewing envelope compared to chewing of the wax cube. As a result, opening angle of masticatory movements for the mixing ability test was greater than for the masticatory performance test. The hardness of test items may be responsible for envelope angle and opening angle. On the contrary, the closing angle near the intercuspal range was found to be related to occlusal guidance. ${ }^{25}$ This may explain why closing angle was similar in the two chewing tests.

In the current study, the mixing ability test and the masticatory performance test showed different masticatory movement patterns. This sug- gests the mixing ability test evaluated partially different aspects of mastication compared to the traditional masticatory performance test. A previous study has shown a significant relationship between MAI and masticatory performance score in dentate subjects. ${ }^{8}$ However, this relationship was not strong $(r=0.66)$, suggesting food mixing ability is partially different from food comminuting ability. The considerable difference in texture of the test items between the mixing ability test and the masticatory performance test is apparently responsible for these differences in chewing results as well as masticatory movements. Thus, clinical application of the mixing ability test in addition to the traditional masticatory performance test is of value to provide further insight into objective masticatory function. On the other hand, it is well known that food type affects not only masticatory movement but also masticatory muscle activity during chewing. ${ }^{13,18,23}$ It would be interesting for the mixing ability test and the masticatory performance test to be compared in respect of masticatory muscle activity.

\section{Conclusion}

In this study, 26 dentate subjects performed the mixing ability test and the masticatory performance test. Masticatory movement pattern regarding range and angle of chewing envelope, velocity, and duration for each phase of chewing cycle, was compared between the two chewing 
tests. The results of this study suggest mandibular movements in the mixing ability test are partially different from those in the masticatory performance test.

Acknowledgments: The authors greatly acknowledge Dr. Neal R. Garrett, University of California Los Angeles, for his valuable contributions. This study was presented at 10th scientific meeting of the Japan Prosthodontic Society Tokyo Chapter.

\section{References}

1. Kimoto K, Ogawa T, Garrett NR et al. Assessment of masticatory performance -Methodologies and their application-. Prosthodont Res Pract 3: 33-45, 2004.

2. Manly RS, Braley LC. Masticatory performance and efficiency. J Dent Res 29: 448-462, 1950.

3. Kapur KK, Soman S, Yurkstas AA. Test foods for measuring masticatory performance of denture wearers. J Prosthet Dent 14: 483-491, 1964.

4. Olthoff LW, van der Bilt A, Bosman F et al. Distribution of particle size in food comminuted by human mastication. Arch Oral Biol 29: 899-903, 1984.

5. Liedberg B, Öwall B. Oral bolus kneading and shaping measured with chewing gum. Dysphagia 10: 101-106, 1995.

6. Hayakawa I, Watanabe I, Hirano S et al. A simple method for evaluating masticatory performance using a color changeable chewing gum. Int J Prosthodont 11: 173-176, 1998.

7. Sato H, Fueki K, Sueda A et al. A new and simple method for evaluating masticatory function using newly developed artificial test food. J Oral Rehabil 30: 68-73, 2003.

8. Sato S, Fueki K, Sato $\mathrm{H}$ et al. Validity and reliability of a newly developed method for evaluating masticatory function using discriminant analysis. J Oral Rehabil 30: 146-151, 2003.

9. Jemt T, Hedegard B, Wickberg K. Chewing pattern before and after treatment with full upper and partial lower free-end dentures. J Prosthet Dent 50: 566-570, 1983.

10. Tallgren A, Mizutani H, Tryde G. A two-year kinesiographic study of mandibular movement patterns in denture wearers. J Prosthet Dent 62: 594-600, 1989.

11. Feine JS, Maskawi K, de Grandmont $\mathrm{P}$ et al. Within-subject comparisons of implant-supported mandibular prostheses: evaluation of masticatory function. J Dent Res 73: 1646-1656, 1994.

12. Dellow PG, Lund JP. Evidence for central timing of rhythmical mastication. J Physiol 215: 1-13, 1971.

13. Lavigne G, Kin JS, Valiquette C et al. Evidence that periodontal pressoreceptors provide positive feedback to jaw closing muscles during mastication. J Neurophysiol 58: 342-358, 1987.

14. Morimoto T, Inoue Y, Nagashima T. Sensory components facilitating Jaw-closing muscle activities in the rabbit. Exp Brain Res 76: 424-440, 1989.

15. Horio T, Kawamura Y. Effects of texture of food on chewing patterns in the human subject. J Oral Rehabil 16: 177-183, 1989.

16. Peyron MA, Mioche L, Renon P et al. Masticatory jaw movement recordings: a new method to investigate food texture. Food qual Pref 7: 229-237, 1996.

17. Plesh O, Bishop B, McCall W. Effect of gum hardness on chewing pattern. J Exp Neurol 92: 502-512, 1986.

18. Peyron MA, Lassauzay C, Woda A. Effects of increased hardness on jaw movement and muscle activity during chewing of visco-elastic model foods. Exp Brain Res 142: 41-51, 2002.

19. Bhatka R, Throckmorton GS, Wintergerst AM et al. Bolus size and unilateral chewing cycle kinematics. Arch Oral Biol 49: 559-566, 2004.

20. Salleh NM, Fueki K, Garrett NR et al. Objective and subjective assessment of hardness of a test item for evaluation of food mixing ability test. J Oral Rehabil 34: 174-183, 2007.

21. Yoshida E, Fueki K, Igarashi Y. Association between food mixing ability and mandibular movements during chewing of a wax cube. J Oral Rehabil 34: 791-799, 2007.

22. Ogawa T, Ogawa M, Koyano K. Different responses of masticatory movements after alteration of occlusal guidance related to individual movement pattern. J Oral Rehabil 28: 830-841, 2001.

23. Foster KD, Woda A, Peyron MA. Effect of texture of plastic and elastic model foods on the parameters of mastication. J Neurophysiol 95: 3469-3479, 2006.

24. Van der Bilt A, Van der Glas HW, Olthoff LW et al. The effect of particles size reduction on the jaw gape in human mastication. J Dent Res 70: 931-937, 1991.

25. Ogawa T, Koyano K, Umemoto G. Inclination of the occlusal guidance as contributing factors in mastication. J Dent 26: 641-647, 1998. 